\title{
Asymptomatic carotid stenosis revascularization: not for all but selected few
}

Volume 6 Issue 5 - 2017

\section{Editorial}

It has been nearly one and a half century since Sir William Richard Gowers, a British neurologist in 1875, linked stroke with extracranial carotid disease. We have come a long way and have accepted the efficacy of carotid revascularization in symptomatic patients as supported by Class I guidelines of America heart association. ${ }^{1}$ There is still great controversy about the management of asymptomatic carotid stenosis (ACS). We here found a distinctive condition where noninvasive, multifaceted best medical treatment is so far not only proving to be a safer approach but also more efficacious than revascularization procedures including carotid endarterectomy or carotid angioplasty and stent placement.

Multicenter randomized studies of carotid endarterectomy (CEA) for carotid artery disease, including Asymptomatic Carotid Artery Stenosis (ACAS) and Asymptomatic Carotid Surgery Trial (ACST) demonstrated a positive statistically significant difference when CEA was utilized, however the absolute risk reduction was rather modest compared to the best medical therapy. ${ }^{2,3}$ The high number of CEAs required to prevent one ipsilateral stroke in combination with recent advances in best medical therapy, has questioned the surgery in asymptomatic patients in providing best clinical practice. ${ }^{4}$ With modern intensive medical therapy, the annual risk of ipsilateral stroke in patients with asymptomatic carotid stenosis is now down to $\leq 1 \%$ per year. ${ }^{5}$ For asymptomatic patients, the risk of ipsilateral stroke after CEA is $0.5 \%$ per year, but this rate may not be significantly lower than that currently associated with medical therapy alone. ${ }^{1}$ When we consider perioperative risks with carotid revascularization procedures in asymptomatic patients, including both carotid endarterectomy and carotid angioplasty and stent placement in major trials including ACAS, ACST, Carotid Revascularization Endarterectomy Versus Stenting Trial (CREST) and The Asymptomatic Carotid Surgery Trial-1 (ACT1), the risk of stroke and death within 30 days ranges from $1.4 \%$ to $3 \%$. Although procedures have become safer over time due to a technological advancement, they are not able to keep up with the efficacy of best medical treatment to a similar extent. We believe that the accepted threshold of $3 \%$ for perioperative complication rates during recanalization procedures may indeed be high given the recent advances in best medical treatment.

There are currently ongoing large, multicenter, international trials in the planning or initial phases to address the key question, is contemporary best medical treatment comparable to carotid revascularization combined with best medical treatment for asymptomatic carotid disease?. Stent-Protected Angioplasty in asymptomatic Carotid artery stenosis versus Endarterectomy Trial-2 (SPACE-2), European Carotid Surgery Trial-2 (ECST-2) and Carotid Revascularization Endarterectomy Versus Stenting Trial-2 (CREST-2) are examples of ongoing trials that include diverse populations across Europe and North America designed to provide the needed evidence to clinicians for the management of asymptomatic carotid artery disease. $^{4}$

\author{
Rakesh Khatri, Anantha R Vellipuram, Alberto \\ Maud, Gustavo J Rodriguez \\ Department of Neurology, Texas Tech University Health \\ Sciences Center of El Paso, USA
}

Correspondence: Gustavo J Rodriguez, MD, Associate Professor and Vice Chair, Neurology Department, Texas Tech University Health Sciences Center of El Paso, Texas, USA, Email rodriguezgustavojose@gmail.com

Received: April 25, 2017| Published: May 02, 2017

Nonetheless it is suggested that there is a subset of high risk for stroke patients among ACS. If appropriately identified they may benefit from carotid revascularization, while others can avoid unnecessary procedures. Several features have been studied and contrary to the popular belief the degree of stenosis alone may not reflect or predict those patients at risk for stroke. Other features based on the morphology of the plaque may be of more concern. The study of plaque morphology including: surface characteristics like ulceration, lipid content of plaque and assessment of the presence of embolic signals on Transcranial Doppler (TCD) seem to provide more useful information for stroke risk stratification. ${ }^{6}$ In the Asymptomatic Carotid Emboli Study (ACES) study, the absolute annual risk of ipsilateral stroke between baseline and 2 years was $3 \cdot 62 \%$ in patients with present embolic signals and $0.70 \%$ in those without. ${ }^{7}$ The combination of embolic signal detection and plaque morphology allows a greater prediction for stroke than either feature alone and identifies a high-risk group with an annual stroke risk of $8 \%$, and a low-risk group of $<1 \%$. This risk stratification may prove useful in the selection of patients with ACS for revascularization procedures. In addition, there is also some evidence that cerebrovascular reactivity as tested with transcranial Doppler $\mathrm{CO} 2$ reactivity, may help with stroke risk stratification in patients with asymptomatic severe carotid artery stenosis or occlusion. ${ }^{8}$ Contralateral symptomatic carotid stenosis and baseline silent infarcts on neuroimaging may also be markers for high stroke risk. Overall about $1 \%$ of the patients with ACS will have a stroke a year and therefore only selected patients, estimated to be around $6 \%$ will require revascularization procedures. ${ }^{6}$ Many trials in our view are mainly concentrated on the degree of stenosis as the main criteria to select high risk patients rather than using other risk stratifying features like TCD to detect emboli, the plaque morphology or proven previous silent brain infarcts on neuroimaging. We agree about the urgent need for trials that compare best medical treatment with carotid revascularization in patients with ACS but additional risk stratifying criteria are needed in order to appropriately identify those 
patients who will benefit from revascularization procedures, patients in whom medical treatment may not be sufficient. Failure to prove benefit of carotid revascularization in selected ACS patients will be deceiving as we certainly know that symptomatic patients with carotid disease were previously asymptomatic. Revascularization procedures in asymptomatic patients should not be offered to all but selected few.

\section{Acknowledgments}

None.

\section{Conflicts of interest}

None.

\section{Funding}

None.

\section{References}

1. Brott TG, Halperin JL, Abbara S, et al. ASA/ ACCF/ AHA/ AANN/ AANS/ ACR/ ASNR/ CNS/ SAIP/ SCAI/ SIR/ SNIS/ SVM/ SVS guideline on the management of patients with extracranial carotid and vertebral artery disease: executive summary. A report of the American College of Cardiology Foundation/American Heart Association Task Force on Practice Guidelines, and the American Stroke Association, American Association of Neuroscience Nurses, American Association of Neurological Surgeons, American College of Radiology, American
Society of Neuroradiology, Congress of Neurological Surgeons, Society of Atherosclerosis Imaging and Prevention, Society for Cardiovascular Angiography and Interventions, Society of Interventional Radiology, Society of NeuroInterventional Surgery, Society for Vascular Medicine, and Society for Vascular Surgery. Circulation. 2011;124(4):489-532.

2. Endarterectomy for asymptomatic carotid artery stenosis. Executive Committee for the Asymptomatic Carotid Atherosclerosis Study. JAMA. 1995;273(18):1421-1428.

3. Halliday A, Harrison M, Hayter E, et al. 10-year stroke prevention after successful carotid endarterectomy for asymptomatic stenosis (ACST-1): a multicentre randomised trial. Lancet . 2010;376(9746):1074-1084.

4. Rubin MN, Barrett KM, Brott TG, et al. Asymptomatic carotid stenosis: What we can learn from the next generation of randomized clinical trials. JRSM Cardiovasc Dis. 2014;3:2048004014529419.

5. Spence JD. Endarterectomy vs. stenting vs. medical therapy. Int $J$ Stroke. 2016;11(5):500-501.

6. Malgor RD WE, Lavarone OA, Labropoulos N. Stratifying risk: asymptomatic carotid disease. J Vasc Bras. 2012;11(1):43-52.

7. Markus HS, King A, Shipley M, et al. Asymptomatic embolisation for prediction of stroke in the Asymptomatic Carotid Emboli Study (ACES): a prospective observational study. Lancet Neurol. 2010;9(7):663-671.

8. Reinhard M, Schwarzer G, Briel M, et al. Cerebrovascular reactivity predicts stroke in high-grade carotid artery disease. Neurology. 2014;83(16):1424-1431. 